\title{
BESICOVITCH MEETS WIENER-FOURIER EXPANSIONS AND FRACTAL MEASURES
}

\author{
ROBERT S. STRICHARTZ
}

1. The evidence. Besicovitch made important contributions to the theory of almost periodic functions and the measure theory of fractal sets. There is a connection between these two areas, in which the almost periodic functions are associated to zero-dimensional measures. Here is the evidence. An almost periodic function on $\mathbf{R}^{n}$ is in essence a function $F(x)$ that can be represented as a series

$$
F(x)=\sum c_{j} e^{i x \cdot a_{j}}
$$

where the amplitudes $c_{j}$ are scalars and the frequencies $a_{j}$ are vectors in $\mathbf{R}^{n}$. (See [B2].) We can regard (1) as a Fourier transform formula $F=(f d \mu)^{\wedge}$ where $\mu$ is counting measure on the set of $a_{j}$ 's and $f\left(a_{j}\right)=c_{j}$. There is a Parseval formula for almost periodic functions,

$$
\lim _{r \rightarrow \infty} \frac{1}{\Omega r^{n}} \int_{B_{r}(y)}|F(x)|^{2} d x=\int|f|^{2} d \mu
$$

where $B_{r}(y)$ denotes the ball of radius $r$ about $y, \Omega$ denotes the volume of the unit ball and $y$ is an arbitrary but fixed point in $\mathbf{R}^{n}$. The left side of (2) is the Bohr mean of $|F|^{2}$.

A well-known theorem of Wiener [W] gives a generalization of (2). Let $\nu$ be any finite measure on $\mathbf{R}^{n}$, and write

$$
\nu=f d \mu+\nu_{c}
$$

for the decomposition into discrete ( $f d \mu$ as above) and continuous $\nu_{c}$ parts. Let $F=\hat{\nu}=\sum c_{k} e^{i x \cdot a_{k}}+\int e^{i x \cdot y} d \nu_{c}(y)$ be the Fourier transform of the measure $\nu$. Then Wiener's theorem says (2) continues to hold. This means that the Fourier transform $\hat{\nu}_{c}$ of the continuous portion of the measure does not contribute to the Bohr mean of $|F|^{2}$. We will interpret Wiener's theorem to mean that every finite measure is in some sense zerodimensional, but the discrete part is the significant zero-dimensional part.

At the other extreme, the $n$-dimensional theory is just the Plancherel formula, which we write

$$
\lim _{r \rightarrow \infty} \int_{B_{r}(y)}|F(x)|^{2} d x=(2 \pi)^{n} \int|f|^{2} d \mu
$$

Received by the editors April 29, 1988.

1980 Mathematics Subject Classification (1985 Revision). Primary 28A75, 42B10; Secondary 42A75, 42B25, 46E35.

Research supported in part by the National Science Foundation, grant DMS-8600245. 
where $d \mu=d x$, Lebesgue measure, and $F(x)=\int e^{i x \cdot y} f(y) d y$, the Fourier transform of $f d \mu$. Doesn't (4) resemble (2)? Another piece of evidence is the formula

$$
\lim _{r \rightarrow \infty} \frac{1}{r} \int_{B_{r}(y)}|F(x)|^{2} d x=2(2 \pi)^{n-1} \int|f|^{2} d \mu
$$

if $d \mu$ is the $n-1$-dimensional surface measure on a sphere, and

$$
F(x)=\int e^{i x \cdot y} f(y) d \mu(y)
$$

This is proved in [Str1], and every closely related results are in [AH].

2. The naive conjectures. It seems that we are looking for a formula of the form

$$
\lim _{r \rightarrow \infty} \frac{1}{r^{n-\alpha}} \int_{B_{r}(y)}|F(x)|^{2} d x=c_{\alpha} \int|f|^{2} d \mu
$$

where $F(x)=\int e^{i x \cdot y} f(y) d \mu(y)$ is the Fourier transform of $f d \mu$ and $\mu$ is some sort of $\alpha$-dimensional measure, for $0 \leq \alpha \leq n$. Now there is a well-known $\alpha$-dimensional measure, called the Hausdorff measure (see Falconer [F] for an extremely readable exposition), which we denote $\mu_{\alpha}$. Since $\mu_{\alpha}$ is not $\sigma$-finite we will usually want to restrict it to a set $E$ so that $\mu_{\alpha \mid E}$ is $\sigma$-finite. The first naive conjecture is that (6) should hold if $\mu=\mu_{\alpha \mid E}$ and $f \in L^{2}(d \mu)$. This would nicely generalize (2), (4), and (5), but it doesn't explain Wiener's theorem. Thus we look for a more general class of $\alpha$-dimensional measures. We say that a Borel measure is locally uniformly $\alpha$-dimensional if

$$
\mu\left(B_{r}(y)\right) \leq c r^{\alpha} \text { for all } r \leq 1 \text { and all } y .
$$

This easily implies that $\mu$ is absolutely continuous with respect to $\mu_{\alpha}$, but since $\mu_{\alpha}$ is not $\sigma$-finite the Radon-Nikodym theorem does not apply. Instead we have the following substitute.

THEOREM 1. $\mu=\varphi d \mu_{\alpha}+\mu^{\prime}$ where $\mu^{\prime}$ has the property $\mu_{\alpha}(A)<\infty$ implies $\mu^{\prime}(A)=0$.

This is the analogue of (3) when $\alpha=0$, so a naive conjecture for the analogue of Wiener's theorem would say

$$
\lim _{r \rightarrow \infty} \frac{1}{r^{n-\alpha}} \int_{B_{r}(y)}|F(x)|^{2} d x=c_{\alpha} \int_{E}|f|^{2} d \mu_{\alpha}
$$

if $\mu=\mu_{\alpha \mid E}+\mu^{\prime}, F=(f d \mu)^{\wedge}$ (for simplicity we take $\varphi=\chi_{E}$, since we can absorb the $\varphi$ into the $f$ ). Although the $\mu^{\prime}$ component becomes negligible in the limit, it will contribute something along the way, so we might also conjecture

$$
\sup _{r \geq 1} \frac{1}{r^{n-\alpha}} \int_{B_{r}(y)}|F(x)|^{2} d x \leq c \int|f|^{2} d \mu .
$$

We will actually be able to establish this last conjecture for all locally uniformly $\alpha$-dimensional measures; the other conjectures will have to be 
substantially modified. However, we should also note that the condition that $\mu$ be locally uniformly $\alpha$-dimensional already represents a retreat from the greatest possible generality. For example, it is not true that $\mu_{\alpha \mid E}$ necessarily satisfies this condition, even if $\mu(E)<\infty$ and even if $E$ satisfies other regularity conditions (see below). But there is a theorem of Besicovitch (see [F]) that asserts that every Borel set $G$ such that $\mu_{\alpha}(G)=+\infty$ contains $E$ such that $0<\mu_{\alpha}(E)<\infty$ and $\mu_{\alpha \mid E}$ is locally uniformly $\alpha$ dimensional; thus there are many examples. When $\alpha=0$ the condition is just that $\mu\left(B_{1}(x)\right)$ be uniformly bounded, so there are some almost periodic functions (whose frequencies have finite limit points) which do not fall into the scope of our results. On the other hand, (9) is not valid for all almost periodic functions.

3. The results. From now on we assume that $\mu$ is a locally uniformly $\alpha$-dimensional measure and $0 \leq \alpha \leq n$.

THEOREM 2. There exists a constant $c$ such that (9) holds for $F=$ $(f d \mu)^{\wedge}$ and all $f \in L^{2}(d \mu)$.

The proof is based on a maximal theorem. Define

$$
m_{\alpha} f(x)=\sup _{0<t \leq 1} t^{-\alpha} \int_{B_{t}(x)}|f| d \mu .
$$

THEOREM 3. The operator $f \rightarrow m_{\alpha} f$ is bounded on $L^{p}(d \mu)$ for $1<p \leq$ $\infty$ and satisfies a weak- $L^{1}(d \mu)$ estimate.

The proof of Theorem 3 is almost a verbatim repeat of one of the proofs of the Hardy-Littlewood maximal theorem [G, pp. 39-43] using the Besicovitch covering lemma which is purely geometric and makes no reference to a measure. To prove Theorem 2 we observe that $f d \mu$ is a tempered distribution so $(f d \mu)^{\wedge}$ is well defined. It is technically easier to deal with Gaussian averages

$$
t^{(n-\alpha) / 2} \int e^{-t|x|^{2}}|F(x)|^{2} d x
$$

as $t \rightarrow 0$, and it is immediate that (9) follows from

$$
\sup _{0<t \leq 1} t^{(n-\alpha) / 2} \int e^{-t|x|^{2}}|F(x)|^{2} d x \leq c \int|f|^{2} d \mu .
$$

The point is that we can write

$$
\begin{aligned}
t^{(n-\alpha) / 2} & \int e^{-t|x|^{2}}|F(x)|^{2} d x \\
& =c t^{-\alpha / 2} \iint e^{-|x-y|^{2} / 4 t} f(x) \overline{f(y)} d \mu(x) d \mu(y)
\end{aligned}
$$

and it is routine to estimate

$$
\left|t^{-\alpha / 2} \int e^{-|x-y|^{2} / 4 t} f(x) d \mu(x)\right| \leq c m_{\alpha} f(y) .
$$

This yields (11) using Theorem 3. 
Concerning the conjectures (6) and (8), we have to give up the hope of obtaining exact identities in most cases and settle for inequalities. First we look at upper bounds.

THEOREM 4. There exist a constant $c$ such that

$$
\limsup _{r \rightarrow \infty} \frac{1}{r^{n-\alpha}} \int_{B_{r}(y)}|F(x)|^{2} d x \leq c \int_{E}|f|^{2} d \mu_{\alpha}
$$

if $\mu=\mu_{\alpha \mid E}+\mu^{\prime}$ is the decomposition in Theorem 1 , and $f \in L^{2}(d \mu)$.

In order to describe lower bounds we need some more definitions. The upper and lower densities $\bar{D}_{\alpha}(\mu, x)$ and $\underline{D}_{\alpha}(\mu, x)$ are defined to be the respective limsup and liminf of $(2 r)^{-\alpha} \mu\left(B_{r}(x)\right)$ as $r \rightarrow 0$. An $\alpha$-dimensional set $E$ is called regular if $\bar{D}_{\alpha}\left(\mu_{\alpha \mid E}, x\right)=\underline{D}_{\alpha}\left(\mu_{\alpha \mid E}, x\right)=1$ for $\mu_{\alpha}$-almost every $x$ in $E$. Regular sets are very rare (in particular, they only occur if $\alpha$ is an integer), so we define a weaker notion of quasi-regular set by the condition $\underline{D}_{\alpha}\left(\mu_{\alpha \mid E}, x\right) \geq c>0$ for $\mu_{\alpha}$-almost every $x$ in $E$. For example, the usual Cantor set is quasi-regular, as are all self-similar fractals.

THEOREM 5. Under the hypotheses of Theorem 4, if in addition $E$ is regular then (8) holds, while if $E$ is only quasi-regular then

$$
\liminf _{r \rightarrow \infty} \frac{1}{r^{n-\alpha}} \int_{B_{r}(y)}|F(x)|^{2} d x \geq c \int_{E}|f|^{2} d \mu_{\alpha}
$$

When $\alpha$ is an integer and $E$ is a $C^{1}$ manifold, these results are essentially contained in $[\mathbf{A H}]$.

The proof of these theorems is again based on (12). As before we reduce the proof to the analogous statements for Gaussian averages. Theorem 2 shows that we may apply the dominated convergence theorem provided we can say something about the limiting behavior of

$$
t^{-\alpha / 2} \int e^{-|x-y|^{2} / 4 t} f(x) d \mu(x) \text { as } t \rightarrow 0 .
$$

But (15) is equal to

$$
\frac{1}{2} t^{-1-\alpha / 2} \int_{0}^{\infty}\left(\int_{B_{r}(y)} f d \mu\right) e^{-r^{2} / 4 t} r d r
$$

by calculus, and we know

$$
\lim _{r \rightarrow 0} \mu\left(B_{r}(y)\right)^{-1} \int_{B_{r}(y)} f d \mu=f(y)
$$

$\mu$-almost everywhere (this is a biproduct of the proof of Theorem 3, and essentially due to Besicovitch [B1]). Thus we expect the behavior of (15) as $t \rightarrow 0$ to be

$$
\frac{1}{2} f(y) \int_{0}^{\infty} r^{-\alpha} \mu\left(B_{r}(y)\right) r^{1+\alpha} t^{-1-\alpha / 2} e^{-r^{2} / 4 t} d r,
$$

which shows clearly the role of the upper and lower densities. Since it is known that $\bar{D}_{\alpha}\left(\mu_{\alpha}, y\right) \leq 1$ for $\mu_{\alpha}$-almost every $y$ in $E[\mathbf{F}]$, and we show 
$\bar{D}_{\alpha}\left(\mu^{\prime}, y\right)=0$ for $\mu_{\alpha}$-almost every $y$ in $\mathbf{R}^{n}$ (again using the Besicovitch covering lemma), the proof of Theorem 4 is essentially complete. The proof of Theorem 5 follows the same ideas, using now the hypotheses on the densities. It is clear that the regularity assumptions in Theorems 4 and 5 are really going to be needed, but the hypotheses that $\mu$ be locally uniformly $\alpha$-dimensional seems less essential. It seems plausible that it can be relaxed. For example, when $\alpha=0$, Besicovitch [B2] shows how to associate an almost periodic function to any set of amplitudes satisfying $\sum\left|c_{j}\right|^{2}<\infty$ and any set of frequencies, such that (2) holds. In the general case there is an immediate problem with the definition of the Fourier transform since $f d \mu$ need not be a tempered distribution. We do have the following result, when $\alpha=0$, strengthening Wiener's theorem but not containing Besicovitch's.

THEOREM 6. Wiener's theorem (2) continues to hold for $F=\hat{\nu}$ and $\nu$ given by (3) provided $\nu$ satisfies

$$
\sum_{k}\left(|\nu|\left(Q_{k}\right)\right)^{2}<\infty
$$

where $\left\{Q_{k}\right\}$ denotes the standard partition of $\mathbf{R}^{n}$ into unit cubes.

These results show that the functions of the form $(f d \mu)^{\wedge}$ have more or less predictable asymptotic behavior. An interesting open question is whether there is some intrinsic characterization of these functions akin to the characterization of almost periodic functions.

Complete proofs are given in [Str2].

\section{REFERENCES}

[AH] S. Agmon and L. Hörmander, Asymptotic properties of solutions of differential equations with simple characteristics, J. d'Analyse Math. 30 (1976), 1-38.

[B1] A. S. Besicovitch, $A$ general form of the covering principle and relative differentiation of additive functions. I, II, and Corrections, Proc. Cambridge Philos. Soc. 41 (1945), 103-110; 42 (1946), 1-10; 43 (1947), 590.

[B2] _ Almost periodic functions, Dover, New York, 1954.

[F] K. J. Falconer, The geometry of fractal sets, Cambridge Univ. Press, 1985.

[G] M. de Guzman, Real variable methods in Fourier analysis, North-Holland, Amsterdam, 1981.

[S] E. M. Stein, Singular integrals and differentiability properties of functions, Princeton Univ. Press, 1970.

[Str1] R. S. Strichartz, Harmonic analysis as spectral theory of Laplacians, J. Funct. Anal. (to appear).

[Str2] _ Fourier asymptotics of fractal measures, preprint. 1933.

[W] N. Wiener, The Fourier integral and certain of its applications, Dover, New York,

Department of Mathematics, White Hall, Cornell University, Ithaca, New YORK 14853 
